Gynäk. Rdsch. 1977;17:I-IV

\title{
Contents, Vol. 17, 1977
}

\section{Nr. 1}

Kurzfassungen von Zeitschriftenartikeln

Kohtad, P. und Klem, V.: Langfristige Nachsorgeüberwachung bei 1121 Fallen von Carcinoma in situ 1

Übersichtsreferat Haldemann, R.; Rohner, A. und Gigon, U.: Das inflammatorische Mammakarzinom .. 9

Mitteilungen der Schweizerischen Gesellschaft fur Gynäkologie

Hormonale Kontrazeption bei Jugendlichen

Ergänzende Resolution über den straflosen Schwangerschaftsabbruch

Communications de la Société Suisse de Gynécologie

Contraception hormonale chez les adolescentes

Resolution complémentaire concernant Tinterruption non-punissable de la grossesse $\quad 58$

Nr. 2

Kurzfassungen von Zeitschriftenartikeln

Sarrut, S.: Maternofetale Listeriose. I. Plazentaveränderungen (75 Fälle) 65

Sarrut, S.; Crumière, C. und Voyer, M.: Maternofetale Listeriose. II. Häufigkeit und Epidemiologie (111 Fälle) 81

Original arbeiten

Dreher, E.; Fürst, M.; Jann, F.X. und Kieliger, F.P.: Ubertritt von Epicillinzum Feten und ins Fruchtwasser sub partu 92

Müller-Tyl, E. und Neufeld, T.: Die Behandlung der vaginalen Trichomoniasis mit Ornidazol 94

Inhaltsverzeichnis IV

Kaufmann, P.: Wie funktioniert die Reihenuntersuchungsstelle der frei praktizierenden Frauenärzte in Bern? 102

Ditesheim, P.J.: La prophylaxie ante partum de la maladie à membranes hyalines du nouveau-né premature $\quad 106$

Schmid, J.: DerEinfluss niederprozentiger Kohlenhydratlösungen auf den mütterlichen und fetalen Stoffwechsel unter der Geburt 114

Nr. 3

Kurzfassungen von Zeitschriftenartikeln

Martin-Comin, J.; Pujol-Amat, P.; Cararach, V.; Davi, E. und Robyn, C: Behandlung der fibrozystischen Erkrankung der Brust mit einem Prolaktin-Inhibitor: 2-Br- $\alpha$ -

Ergokryptin (CB-154) 121

Pearlman, N.W.; Guerra, O. und Fracchia, A.: Primär inoperabler Brustkrebs 126

Stocks, L.H. und Simmons Patterson, F.M.: Inflammatorisches Brustkarzinom 132 
Schwartz, G.F.; Feig, S.E.; Libschitz, H.I.; Patchefsky, A.S.; Nerlinger, R.E. und Wallace, J.D.: Reihen-Vorsorgeuntersuchungen auf Brustkrebs (Ergebnisse, Probleme, Erwartungen) 135

Original arbeiten

Tulzer, H. und Kupka, St.: Über den Unterschied der Effektivität der Lymphonodektomie bei der Radikaloperation des Kollumkarzinoms mit und ohne Drüsenbefall . 141 Gerber, H. und Baur, B.: Therapie mit Anthelmintika während der Schwangerschaft . .

Keller, P.J.: Die Therapie dergestörten Ovarialfunktion. Einfúhrung

Gigon, U.: Clomiphenund Cyclofenil

Keller, P.J.: Die Therapie der gestörten Ovarialfunktion. LH-RH

Bihrer, R.; Floersheim, Y. und Keller, P.J.: Hypergonadotrope Amenorrhö

Haldemann, R. und Gigon, U.: Zur Frage der Proteo- und Steroidhormonausscheidung unter Clomiphenbehandlung 186

Suter, H.R.; Floersheim, Y. und Keller, P.J.: Uberwachung der Clomiphenbehandlung durch Serumöstradiolbestimmungen 190

Nr. 4

Herrn Professor Dr. med. Horst Schwalm zum Gedenken

193

Systematisches Gesamtregister der Volumen 1-17

Zusammengestellt von H. Schwalm $\uparrow$, ergänztvonM. Berger

Supplementum

Schweizerische Gesellschaft für Gynäkologie

Bericht über die Jahresversammlung, 30. Juni-2. Juli 1977 in Lugano

Société Suisse de Gynécologie

Compte rendu de $\Gamma$ Assemblée annuelle, 30 juin-2 juillet 1977 à Lugano

$\notin \mathrm{p}$

$1 / 8 \mathrm{tf}<3 / 4$

,$<\mathrm{fc} \theta$ 